\title{
Tuber pulchrosporum sp. nov., a black truffle of the Aestivum clade (Tuberaceae, Pezizales) from the Balkan peninsula
}

\author{
Elias Polemis', Georgios Konstantinidis², Vassiliki Fryssouli', Monica Slavova ${ }^{3}$, \\ Triantafyllos Tsampazis ${ }^{4}$, Vasileios Nakkas ${ }^{5}$, Boris Assyov ${ }^{6}$, Vasileios Kaounas ${ }^{7}$, \\ Georgios I. Zervakis'
}

I Agricultural University of Athens, Laboratory of General and Agricultural Microbiology, Iera Odos 75, 11855 Athens, Greece 2 Agiou Kosma 25, 51100 Grevena, Greece 34 Krivolak Street, Sofia, 1000, Bulgaria 4 Aristotelous 106, Maniaki, 52100 Kastoria, Greece 5 Konstantinou Davaki 218, Neochoropoulo, 45500 Ioannina, Greece 6 Institute of Biodiversity and Ecosystem Research, Bulgarian Academy of Sciences, 2 Gagarin Str, Sofia, 1113, Bulgaria 7 Sokratous 40, 19016 Artemis, Attica, Greece

Corresponding author: Georgios I. Zervakis (zervakis@aua.gr)

Academic editor: T. Lumbsch | Received 3 December 2018 | Accepted 7 January 2019 | Published 20 February 2019

Citation: Polemis E, Konstantinidis G, Fryssouli V, Slavova M, Tsampazis T, Nakkas V, Assyov B, Kaounas V, Zervakis GI (2019) Tuber pulchrosporum sp. nov., a black truffle of the Aestivum clade (Tuberaceae, Pezizales) from the Balkan peninsula. MycoKeys 47: 35-51. https://doi.org/10.3897/mycokeys.47.32085

\begin{abstract}
Knowledge on the diversity of hypogeous sequestrate ascomycetes is still limited in the Balkan Peninsula. A new species of truffle, Tuber pulchrosporum, is described from Greece and Bulgaria. Specimens were collected from habitats dominated by various oak species (i.e. Quercus ilex, Q. coccifera, Q. robur) and other angiosperms. They are morphologically characterised by subglobose, ovoid to irregularly lobed, yellowish-brown to dark brown ascomata, usually with a shallow basal cavity and surface with fissures and small, dense, almost flat, trihedral to polyhedral warts. Ascospores are ellipsoid to subfusiform, uniquely ornamented, crested to incompletely reticulate and are produced in (1-)2-8-spored asci. Hair-like, hyaline to light yellow hyphae protrude from the peridium surface. According to the outcome of ITS rDNA sequence analysis, this species forms a distinct well-supported group in the Aestivum clade, with T. panniferum being the closest phylogenetic taxon.
\end{abstract}

\section{Keywords}

Ascomycota; Tuberaceae; truffle; ectomycorrhizal fungi; taxonomy; phylogeny; fungal diversity

Copyright Elias Polemis et al. This is an open access article distributed under the terms of the Creative Commons Attribution License (CC BY 4.0), which permits unrestricted use, distribution, and reproduction in any medium, provided the original author and source are credited. 


\section{Introduction}

The genus Tuber F.H. Wigg. (Ascomycota, Pezizales, Tuberaceae) is globally famous and historically appreciated for the production of hypogeous ascomata, known as 'truffles'; several of them are highly prized due to their unique aroma and culinary value. Moreover, the genus is known for the symbiotic ectomycorrhizal associations that its members form with several gymnosperm and angiosperm forest-tree species as well as with orchids (Riousset et al. 2001; Selosse et al. 2004; Mello et al. 2006; Trappe et al. 2009). Furthermore, truffles are also important for serving as a primary or supplementary source of nutrition for soil micro-fauna and several mammals (Hanson et al. 2003; Trappe and Claridge 2010; Schickmann et al. 2012).

A continuous interest in the study of this particular group has resulted in several recent reports on new Tuber species from various parts of the world (e.g. Crous et al. 2017; Fan et al. 2015; Guevara-Guerrero et al. 2018; Pińa Páez et al. 2018). It is estimated that their number ranges between 180 and 220 (Zambonelli et al. 2016) nested in 11 major phylogenetic clades (Bonito et al. 2013). In particular, the Aestivum clade is composed of species associated with a large spectrum of host plants and are reported to occur in the Old World, i.e. Europe, North Africa and/or Asia (Jeandroz et al. 2008; Bonito et al. 2013; Payen et al. 2014). Indicative examples are T. aestivum Vittad. (the type species of the genus), T. panniferum Tul. \& Tul., T. malenconii Donadini, Riousset, G. Riousset \& G. Chev. and T. mesentericum Vittad., as well as T. sinoaestivum Zhang \& Liu recently described from China (Zambonelli et al. loc. sit.; Zhang and Chen 2012). The morphologically diverse and economically important species T. magnatum Picco also forms part of this clade (Bonito et al. 2010a; 2013).

Although Tuber diversity is well documented in Europe (Bonito at al. 2010a, Ceruti et al. 2003, Jeandroz et al. 2008), the south-eastern part of the continent and especially the Balkan Peninsula was until recently poorly investigated. Indicative of this fact is that, by the end of the last century, only three Tuber species had been recorded in Greece (Zervakis et al. 1999). However, during the last two decades, an ever increasing interest in the collection of truffles led to a remarkable increase in the number of pertinent records (e.g. Diamandis and Perlerou 2008; Konstantinidis 2009; Agnello and Kaounas 2011; Alvarado et al. 2012a,b; Gyosheva et al. 2012); thus, to date, 15 Tuber spp. are reported from Greece. Similarly, only two Tuber spp. had been recorded in Bulgaria by the end of the last century; however, this number is fast-growing during the last few years and 14 species are currently known to exist (Dimitrova and Gyosheva 2008; Gyosheva et al. 2012; Lacheva 2012; Nedelin et al. 2016; Assyov and Slavova 2018). Regarding adjacent countries, 12 truffle species were reported to occur in Serbia, including one recently described (Marjanović et al. 2010; Milenković et al. 2015), while six Tuber spp. were recorded in Montenegro, five in FYROM and four in Albania (Pacioni 1984; Marjanović et al. 2010).

In the frame of this work, several truffle specimens originating from north and central continental Greece and from Bulgaria were studied with respect to their morphology and phylogenetic relationships to other Tuber taxa and a new species is hereby proposed. 


\section{Methods}

\section{Sampling and Morphological characterisation}

Specimens used for this study were collected during 2008-2017 from north and central Greece (Regions of Epirus, Thessaly, Eastern Macedonia and Thrace, Western Greece and Attica), as well as from Bulgaria (Regions of Eastern Stara Planina and Black Sea coast). Specimens are deposited in the fungaria of the Laboratory of General and Agricultural Microbiology (Agricultural University of Athens, ACAM), of the Institute of Biodiversity and Ecosystem Research (SOMF) and the authors' personal collections. Macroscopic characters such as size, peridium surface texture, colour and odour were observed in fresh ascomata. Colour coding and terminology is derived from the "Flora of British Fungi - Colour Identification Chart" (Royal Botanic Garden Edinburgh 1969).

Microscopic characters were examined by hand-cut sections on fresh and dried material, using a Zeiss Axioimager A2 microscope under bright field and Differential Interference Contrast (DIC) and an AmScope T360B. Microphotographs were taken with the aid of a mounted digital camera (Axiocam). Microscopic observations were performed in water, $3 \%(\mathrm{w} / \mathrm{v})$ potassium hydroxide $(\mathrm{KOH})$ and Melzer's reagent. To assess the ascospore size, a minimum of 30 mature ascospores from each type of asci ( 2 to 8 -spored) were measured and dimensions are provided as (minimum) average \pm standard deviation (maximum); quotient (Q), i.e. length divided by the width, was calculated for each ascospore and the median value $(\mathrm{Qm})$ is given. For scanning electron microscopy (SEM), ascospores were scraped from the hymenial surface and mounted on aluminium foil, which was then fixed on a microscope holder and sputter-coated with gold. Observations were performed in JEOL JSM-5510.

\section{DNA sequencing and Phylogenetic analyses}

Total genomic DNA was extracted from herbarium specimens using the Nucleospin Plant II DNA kit (Macherey and Nagel, Germany) following the manufacturer's protocol with minor modifications. The internal transcribed spacer (ITS) region of nuclear ribosomal DNA (nrDNA) was amplified using the primer combination ITS1/ ITS4 (White et al. 1990). Polymerase chain reactions (PCR) were performed in $50 \mu \mathrm{l}$ containing $50 \mathrm{ng}$ DNA template, $0.25 \mu \mathrm{M}$ of each primer, $0.2 \mathrm{mM}$ of each dNTP, $1 \times$ HiFi Buffer (Takara BIO INC., Japan) and 1 U HiFi Taq DNA polymerase (Takara BIO INC., Japan). Conditions for PCR amplification were as follows: $94^{\circ} \mathrm{C}$ for $5 \mathrm{~min}$, followed by 35 cycles of $94^{\circ} \mathrm{C}$ for $30 \mathrm{sec}, 50^{\circ} \mathrm{C}$ for $30 \mathrm{sec}$ and $72^{\circ} \mathrm{C}$ for $1 \mathrm{~min}$, with a final extension at $72^{\circ} \mathrm{C}$ for $10 \mathrm{~min}$. PCR products were purified using Invitrogen PureLink kit (Thermo Fisher Scientific, Korea) and were submitted for sequencing to CeMIA SA (Larissa, Greece). DNA sequences were then visualised, manually edited and assembled using UGENE (Okonechnikov et al. 2012). Validated sequences, generated in this study, were deposited in GenBank under the accession numbers MK113975 to 
Table I. Details of ITS sequences deriving from Tuber pulchrosporum sp. nov. and from reference material used for the construction of the phylogenetic tree. Clades names are placed in the order they appear in Fig. 5.

\begin{tabular}{|c|c|c|c|c|}
\hline Species/ Clade & Collection code & $\begin{array}{c}\text { GenBank } \\
\text { Accession No. }\end{array}$ & Origin & Reference \\
\hline \multicolumn{5}{|l|}{ Excavatum Clade } \\
\hline \multirow[t]{2}{*}{ Tuber fulgens } & M2435 & HM485358 & Italy & Bonito et al. 2010a \\
\hline & HMT37 & HM151976* & Austria & Urban et al. 2010 \\
\hline \multirow[t]{2}{*}{ Tuber excavatum } & SA1TE & $\mathrm{KJ} 524533^{*}$ & Poland & Hilszczanska et al. 2014 \\
\hline & JST62014 & KX354295 & Germany & Schiebold et al. 2017 \\
\hline \multicolumn{5}{|l|}{ Gennadii Clade } \\
\hline \multirow[t]{2}{*}{ Tuber lacunosum } & AH39255 & JN392212 & Spain & Alvarado et al. 2012a \\
\hline & АH38932 & JN392213 & Spain & Alvarado et al. 2012a \\
\hline \multirow[t]{4}{*}{ Tuber gennadii } & B M1904 & HM485361 & Italy & Bonito et al. 2010a \\
\hline & AH39251 & JN392211 & Spain & Alvarado et al. 2012a \\
\hline & AH31113 & JN392203 & Spain & Alvarado et al. 2012a \\
\hline & AH38957 & JN392204 & Spain & Alvarado et al. 2012a \\
\hline \multicolumn{5}{|l|}{ Regianum Clade } \\
\hline \multirow[t]{2}{*}{ Tuber bernardinii } & 2172 & KY420104 & Italy & Merenyi et al. 2017 \\
\hline & NA & KY420105 & Italy & Merenyi et al. 2017 \\
\hline \multirow[t]{2}{*}{ Tuber magentipunctatum } & MO793 & KY420089 & Italy & Merenyi et al. 2017 \\
\hline & ZB4293 & JQ288909** & Hungary & Merenyi et al. 2017 \\
\hline \multirow{2}{*}{ Tuber regianum } & ZB3081 & KY420098 & Slovakia & Merenyi et al. 2017 \\
\hline & erd-2590 & KY420102 & Spain & Merenyi et al. 2017 \\
\hline \multicolumn{5}{|l|}{ Macrosporum Clade } \\
\hline \multirow[t]{2}{*}{ Tuber macrosporum } & Macro1 & AF106885* & Italy & Rubini et al. 1998 \\
\hline & HMSFI_TUBMAC/141207A & FM205634* & Slovenia & Grebenc et al. 2008 \\
\hline \multicolumn{5}{|l|}{ Aestivum Clade } \\
\hline \multirow[t]{2}{*}{ Tuber magnatum } & JT19460 & HM485374 & Italy & Bonito et al. 2010a \\
\hline & GB12 & JQ925645 & Italy & Bonito et al. 2013 \\
\hline \multirow[t]{2}{*}{ Tuber malenconii } & MA:Fungi:28384/ 02MLC & FM205597 & Spain & Grebenc et al. 2008 \\
\hline & 17110 & JF908743 & Italy & Osmundson et al. 2013 \\
\hline \multirow[t]{2}{*}{ Tuber sinoaestivum } & L4213 & KY081688* & & Wang and Wang 2016 \\
\hline & JP-Zhang-140 & JN896355 & China & Zhang et al. 2012 \\
\hline \multirow[t]{2}{*}{ Tuber aestivum } & TaeW016I-E134 & AJ888090 & Italy & Weden 2005 \\
\hline & S19 & HQ706002 & Slovakia & Gryndler et al. 2011 \\
\hline \multirow[t]{2}{*}{ Tuber uncinatum } & MA: Fungi: 24605 & FM205618* & Spain & Grebenc et al. 2008 \\
\hline & 228 & AJ492199 & Italy & Mello et al. 2002 \\
\hline \multirow[t]{2}{*}{ Tuber mesentericum } & CW105 & HM485375 & Sweden & Bonito et al. 2010a \\
\hline & UASWS1612 & KY197989* & Switzerland & Cochard et al. 2016 \\
\hline \multirow[t]{2}{*}{ Tuber panniferum } & - & AF132507 & & Roux et al. 1999 \\
\hline & JT12835 & HM485380 & Spain & Bonito et al. 2010a \\
\hline \multirow{8}{*}{$\begin{array}{l}\text { Tuber pulchrosporum } \\
\text { sp. nov. }\end{array}$} & $1945 \mathrm{~F} 8517$ & MK113981 & Bulgaria & This work \\
\hline & 1961 F0388 & MK113982 & Bulgaria & This work \\
\hline & VN091 (holotype) & MK113975 & Greece & This work \\
\hline & GK3801 & MK113979 & Greece & This work \\
\hline & LT1183 & MK113976 & Greece & This work \\
\hline & GK9408 & MK113977 & Greece & This work \\
\hline & VK4482 & MK113980 & Greece & This work \\
\hline & GK6538 & MK113978 & Greece & This work \\
\hline Multimaculatum Clade & & & & \\
\hline Tuber multimaculatum & OSC 62169 & HM485377 & Spain & Bonito et al. 2010a \\
\hline Rufum Clade & & & & \\
\hline Tuber rufum & 1785 & EF362475 & Italy & Iotti et al. 2007 \\
\hline & $S 90$ & JF926123 & Germany & Stobbe et al. 2012 \\
\hline
\end{tabular}




\begin{tabular}{|c|c|c|c|c|}
\hline Species/ Clade & Collection code & $\begin{array}{c}\text { GenBank } \\
\text { Accession No. }\end{array}$ & Origin & Reference \\
\hline \multicolumn{5}{|l|}{ Melanosporum Clade } \\
\hline \multirow[t]{2}{*}{ Tuber pseudoexcavatum } & T14_HKAS44325b & GU979039 & China & Chen et al. 2011 \\
\hline & Tpse-yn05 & DQ329374 & China & Wang et al. 2006 \\
\hline Tuber regimontanum & ITCV 909 & EU375838 & Mexico & Guevara et al. 2008 \\
\hline \multirow[t]{2}{*}{ Tuber indicum } & Ascocarpe I1 & AF300822 & China & Mabru et al. 2001 \\
\hline & HKAS 39501 & AY514305 & China & Zhang et al. 2005 \\
\hline \multirow[t]{2}{*}{ Tuber melanosporum } & SB2-6 & MF693845 & France & $\begin{array}{c}\text { Schneider-Maunoury et } \\
\text { al. } 2018\end{array}$ \\
\hline & P_Qr & KP972070 & Canada & Berch and Bonito 2016 \\
\hline \multicolumn{5}{|l|}{ Tumericum Clade } \\
\hline \multirow[t]{2}{*}{ Tuber turmericum } & BJTC FAN475 & KT758839 & China & Fan et al. 2015 \\
\hline & BJTC FAN473 & KT758837 & China & Fan et al. 2015 \\
\hline \multicolumn{5}{|l|}{ Gibbosum Clade } \\
\hline Tuber oregonense & DUKE GB284 & FJ809874 & USA & Bonito et al. 2010b \\
\hline Tuber gibbosum & OSC 40964 & FJ809863 & USA & Bonito et al. 2010b \\
\hline \multicolumn{5}{|l|}{ Maculatum Clade } \\
\hline \multirow[t]{2}{*}{ Tuber maculatum } & A15 & AM406673 & Italy & El Karkouri et al. 2007 \\
\hline & Db-A & MH040280* & & Sikora 2018 \\
\hline \multicolumn{5}{|l|}{ Latisporum Clade } \\
\hline Tuber latisporum & HKAS 44315 & DQ898183 & China & Chen and Liu 2007 \\
\hline Tuber & BJTC Fan 250 & KF744063 & China & Fan and Yue 2013 \\
\hline \multicolumn{5}{|l|}{ pseudosphaerosporum } \\
\hline \multicolumn{5}{|l|}{ Puberulum Clade } \\
\hline Tuber cistophilum & AH 39275 & JN392231 & Spain & Alvarado et al. 2012a \\
\hline Tuber borchii & Tar042 & KT165326 & Italy & Belfiori et al. 2016 \\
\hline \multirow[t]{2}{*}{ Tuber sphaerospermum } & AH38930 & JN392244 & Morocco & Alvarado et al. 2012a \\
\hline & AH39190 & JN392246 & Spain & Alvarado et al. 2012a \\
\hline \multicolumn{5}{|l|}{ Outgroup } \\
\hline \multirow[t]{2}{*}{ Choiromyces alveolatus } & 22830 & AF501258 & & Ferdman et al. 2005 \\
\hline & p612i & EU697268* & & Gordon 2008 \\
\hline
\end{tabular}

* unpublished sequence.

** this sequence appears as "T. regianum" in GenBank (unpublished; Merenyi et al. 2011).

MK113982 (Table 1). Moreover, the percent sequence identity was estimated by using ClustalOmega (Sievers and Higgins 2018) through the EMBL-EBI portal.

A total of 62 Tuber ITS rDNA sequences were used for phylogenetic analysis by including eight sequences of T. pulchrosporum sp. nov. and 54 sequences from GenBank (nine of them representing type specimens) which correspond to 31 Tuber taxa mainly of European distribution (Table 1). Choiromyces alveolatus (Harkn.) Trappe (AF501258, EU697268) was used as the outgroup. Sequence alignment was performed through the online version of the multiple sequence alignment programme MAFFT v7 (Katoh and Standley 2013) by applying the Q-INS-I strategy and alignments were inspected and manually adjusted at misaligned sites by using MEGAX (Kumar et al. 2018). The pertinent matrix was deposited in TreeBASE under the accession number 23587.

Phylogenetic relationships of taxa were inferred by using maximum likelihood (ML) and Bayesian Inference (BI) through the CIPRES portal (www.phylo.org; Miller et al. 2010). ML analysis of the ITS dataset was conducted by RAxML v8.2 (Stama- 
takis 2014) with 1,000 bootstrap replicates and search for the best-scoring ML tree. BI analysis was performed by MrBayes v3.2.1 (Ronquist et al. 2012) and the General Time Reversible + Gamma $(G T R+G)$ model was selected as the best model under the Akaike Information Criterion (AIC) implemented in MrModeltest v2.3 (Nylander 2004). To estimate posterior probabilities, 20,000,000 Markov chain Monte Carlo (MCMC) simulation generations were run in two parallel independent runs of four chains, one cold and three heated, with trees sampled every 1,000 generations and the first 25\% of trees were omitted as burn-in. A 50\% majority rule consensus tree was built and visualised with iTOL (Letunic and Bork 2016). Clades with bootstrap support (BS) $\geq 70 \%$ and Bayesian posterior probability (PP) $\geq 95 \%$ were considered as significantly supported.

\section{Results}

Taxonomy

\section{Tuber pulchrosporum Konstantinidis, Tsampazis, Slavova, Nakkas, Polemis, Frys- souli \& Zervakis, sp. nov.}

MycoBank: MB 828883

GenBank: MK113975

Fig. 1a

Type. GREECE. Ioannina Prefecture: Ioannina city, 39³6'39"N, 2050'05"E, 500 m alt., in soil under a pure stand of Quercus coccifera L., 27 Apr 2016, coll. V. Nakkas, VN091, holotype: ACAM 2016-007 (ACAM!); isotype: SOMF 29980 (SOMF!).

Diagnosis. Ascomata $0.6-7(-10) \mathrm{cm}$ in diam., subglobose, ovoid to irregularly lobed, usually with shallow basal cavity, surface with fissures and small, dense, almost flat trihedral to polyhedral warts, yellowish-brown to dark brown. Ascospores 25.0 $37.0 \times 18.2-25.6 \mu \mathrm{m}$ in (1-)2-8-spored asci, ellipsoid to subfusiform on average, $\mathrm{Qm}=1.4$, crested to incompletely reticulate. Hair-like, hyaline to light yellow-brown hyphae protruding from peridium surface.

T. panniferum, the closest phylogenetically-related species, produces smaller ascospores $(23-26 \times 18-20 \mu \mathrm{m})$, broadly ellipsoid to subglobose on average, with isolated warts; moreover, the peridium surface is woolly-felted due to the presence of dense rusty brown hair-like hyphae.

Etymology. "pulchrosporum" refers to the uniquely distinct/impressive ornamentation of the ascospores.

Description. Ascomata 0.6-7(-10) cm in diameter, tuberous, subglobose, ovoid to irregularly lobed, usually depressed with a shallow - occasionally prominent - basal cavity (excavated), covered up with whitish to yellowish rhizomorphs, fragile, initially greyish to yellowish-brown [fawn (29), sienna (11), fulvous (12)], darkening in maturity to brown [snuff brown (17), umber (18), bay (19), to date brown (24)] or with 


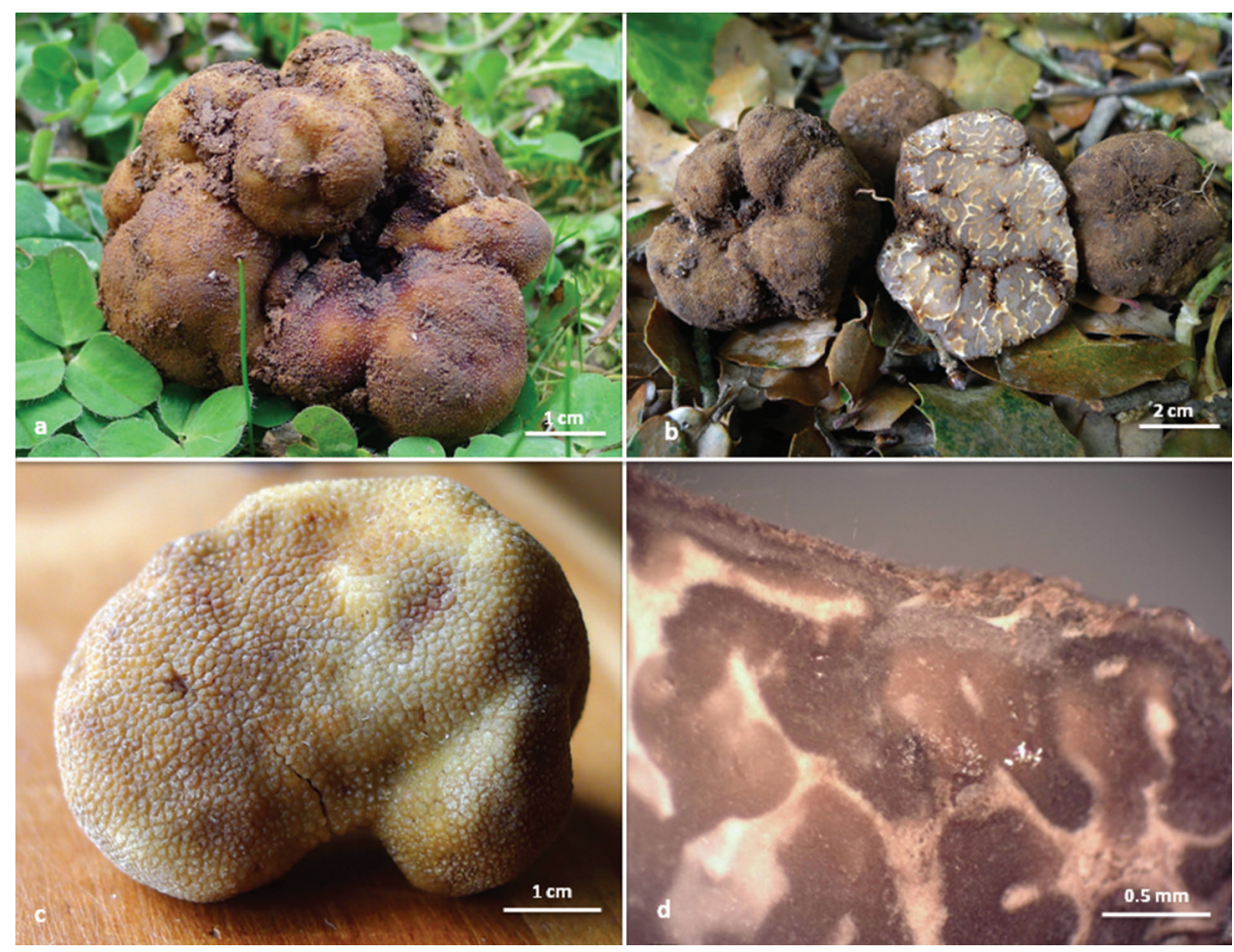

Figure I. T. pulchrosporum sp. nov.: a ascomata in situ (holotype) b ascomata in situ (paratype) c detail of peridium surface (paratype) $\mathbf{d}$ section of peridium (paratype).

some shades of purple tinges [purplish date (22), purplish chestnut (21) to brown vinaceous (25)], sometimes with darker black [fuscous black (38)] spots, surface rarely almost smooth, usually rough, with fissures and small, dense, almost flat trihedral to polyhedral warts. Gleba with one of more cavities, initially pinkish-grey [vinaceous buff (31), clay pink (30)], then greyish-brown [milky coffee (28)], yellowish-brown [fulvous (12)], brown [snuff brown (17), umber (18), bay (19)], to purplish-brown in maturity [purplish date (22) to purplish chestnut (21)], with bay (19) to rusty tawny (14) coloured areas close to the cavity, marbled with relatively few and thick white veins, that sometimes are reddening (Fig. 1). Odour pleasant truffle-like.

Peridium 120-370 $\mu \mathrm{m}$ thick, consisting of two layers; the outer layer 50-160 $\mu \mathrm{m}$ thick, pseudoparenchymatous, composed of yellowish-brown and subglobose inwards to subangular dark brown cells outwards; 4.0-16.3 × 2.5-13.2 $\mu \mathrm{m}$, thick-walled (1.5$2.5 \mu \mathrm{m}$ ); the inner layer 70-210 $\mu \mathrm{m}$, composed of pale yellow or hyaline and thickwalled, interwoven hyphae, $2-10 \mu \mathrm{m}$ in diameter, forming an intricate texture, becoming agglutinated when dried. Surface with abundant isolated, hyaline to golden-yellow (in water or $\mathrm{KOH}$ ), thick-walled hair-like hyphae (walls 1.0-1.5 $\mu \mathrm{m}$ ), 30-140 $\mu \mathrm{m}$ long (occasionally exceeding $300 \mu \mathrm{m}$ in Bulgarian specimens) and $2.5-4.5 \mu \mathrm{m}$ broad at base, 1-2 septate (Figs 1, 2). 


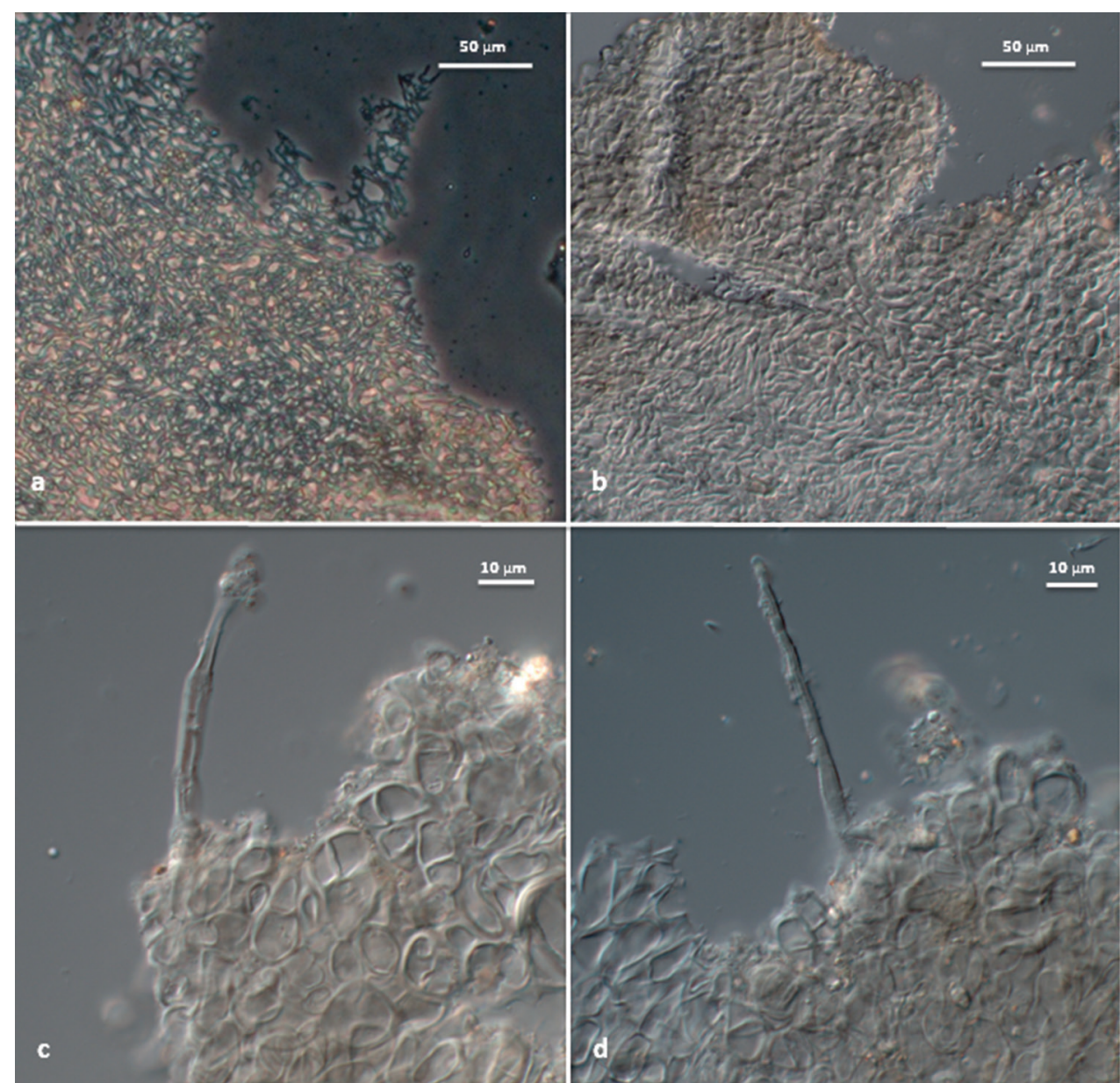

Figure 2. T. pulchrosporum sp. nov.: a, b peridium structure $\mathbf{c}, \mathbf{d}$ hair-like hyphae on peridium surface.

Ascospores hyaline when young then yellowish, yellow-brown to brown, at most ellipsoid to subfusiform, some broadly ellipsoid, subglobose to globose, rarely almost limoniform in initial stages, thin-walled and smooth when young, becoming thickwalled at maturity, walls $2-3.5(-4) \mu \mathrm{m}$ thick, usually crested to incompletely reticulate, measured (excluding the ornamentation) in the rare 1-spored asci (28-) 46.7 \pm 7.4 $(-57) \times(20-) 29.4 \pm 4.6(-34) \mu \mathrm{m}$, in 2-spored asci (27-) $39.5 \pm 5.8(-53) \times(21-)$

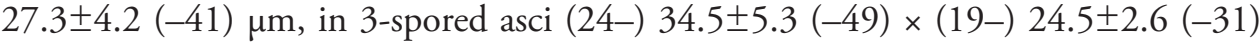
$\mu \mathrm{m}$, in 4-spored (21-) 30.9 $\pm 4.9(-39) \times(18-) 22.2 \pm 2.7(-30) \mu \mathrm{m}$, in 5-spored asci

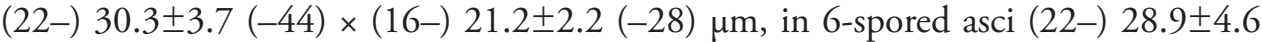
$(-37) \times(17-) 20.6 \pm 2.0(-28) \mu \mathrm{m}$, in 7 -spored asci $(21-) 27.8 \pm 3.3(-35) \times(13-)$ $19.9 \pm 2.7(-27) \mu \mathrm{m}$ and in 8-spored asci (20-) $25.4 \pm 2.6(-31) \times(14-) 18.4 \pm 3.1$ (-26) $\mu \mathrm{m}$ (Fig. 3); Q=1.0-2.2, Qm=1.43 \pm 0.19 ; ornamentation with (0-) $1-2(-4)$ thick veins across the long axis with few to several transverse outgrowths, rarely al- 


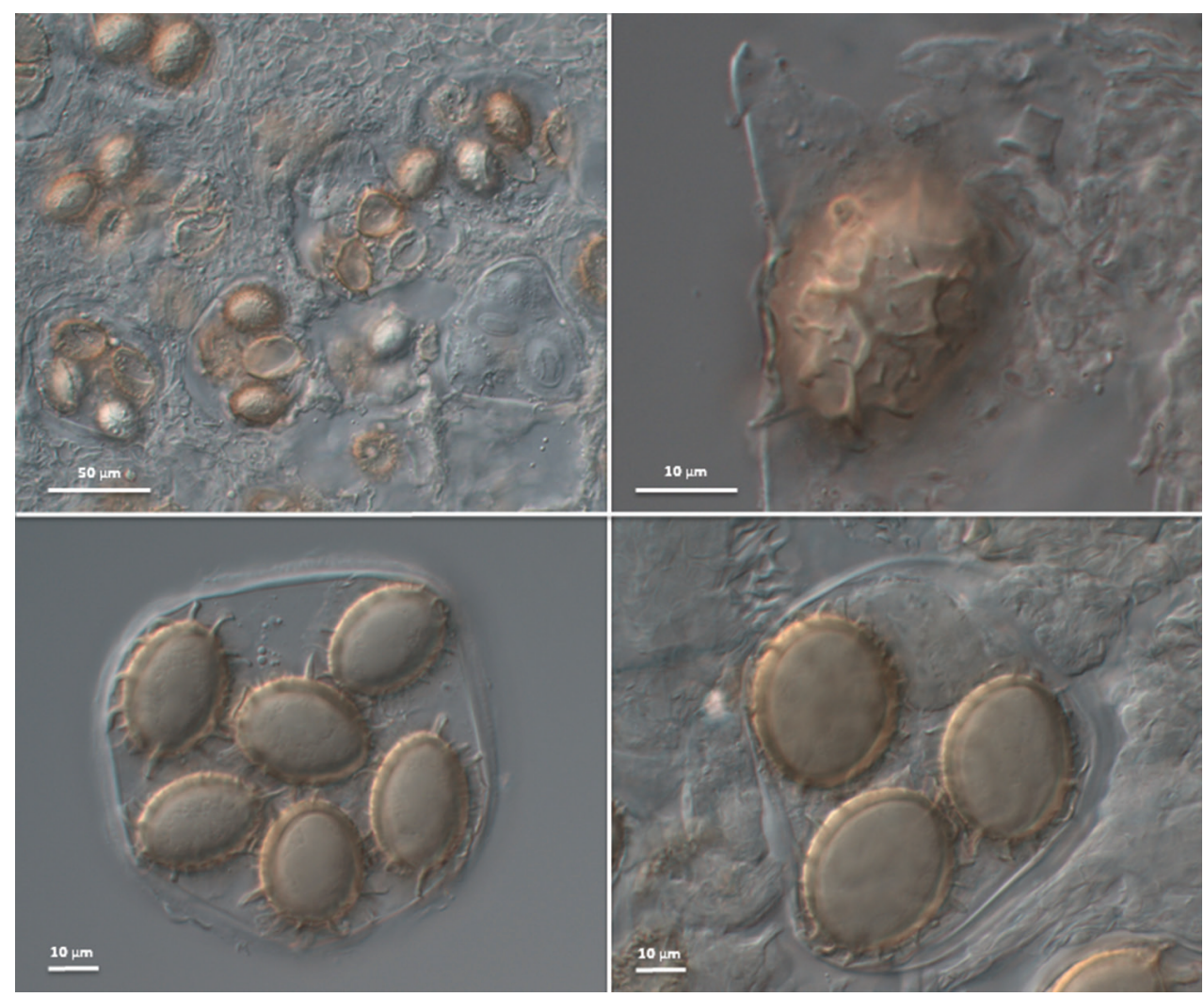

Figure 3. T. pulchrosporum sp. nov.: asci and ascospores.

most completely reticulate in maturity and then with (0-)2-10(-15) meshes in the longitudinal direction; circumferentially with 22-42 conical warts, with pointed or blunt, straight or curved apices, rarely forked, 1.5-6(-8) $\mu \mathrm{m}$ tall (Fig. 4); not reacting with Melzer's reagent. Asci (64-) 78-96 (-121) × (50-) 65-84 (-98) $\mu \mathrm{m}$ (excluding stalk), globose, subglobose, ellipsoid, rarely subangular, with a short stalk, 6.5-9(-15) $\times 6.5-7.5(-10.5) \mu \mathrm{m},(1-) 2-8$-spored (Fig. 3).

Distribution and ecology. Hypogeous, in soil, appearing solitary or in small groups from March to June, under Quercus sp., Q. coccifera or Q. ilex L. or under Carpinus sp. or in mixed stands of Quercus sp. and Pinus nigra J.F. Arnold or of Q. ilex and Pinus halepensis Miller or of Quercus robur L., Corylus sp., Carpinus sp. and Acer sp. It seems to be rather common in continental (northern and central) Greece, while it also occurs in the regions of Eastern Stara Planina and the Black Sea coast of Bulgaria.

Additional collections examined (paratypes). GREECE. Xanthi Prefecture: Toxotes, in soil under a mixed stand dominated by Q. coccifera, 20 June 2008, GK3186b (ACAM 2010-127), coll. P. Panagiotidis. Aitoloakarnania Prefecture: Xiromero, in soil under pure forest of Quercus sp., 10 May 2009, GK3801 (ACAM 2010-129), coll. Ch. Chrysopoulos and K. Giatra (GenBank: MK113979); Xiromero, in soil under pure for- 


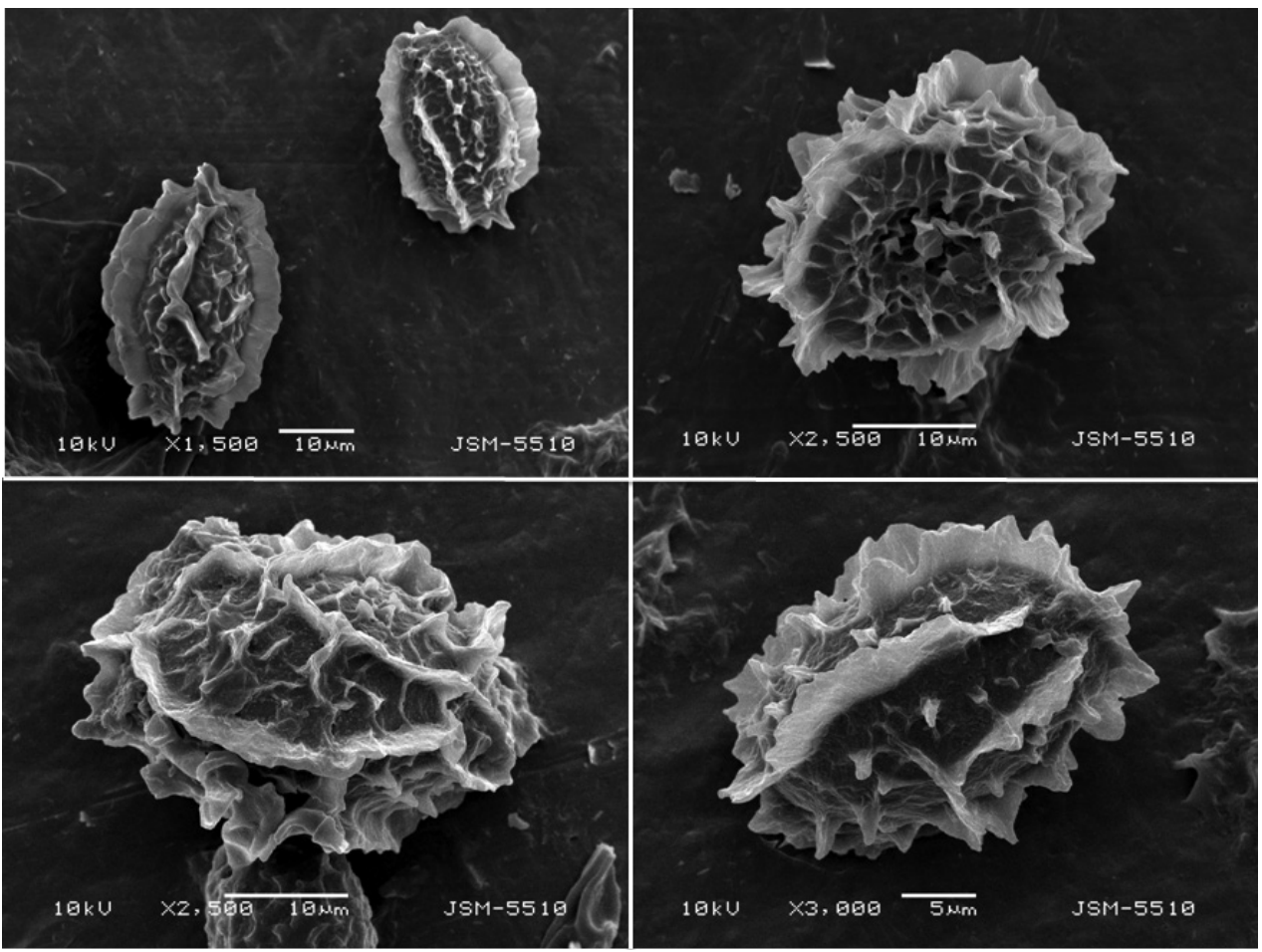

Figure 4. T. pulchrosporum sp. nov.: SEM of ascospores.

est of Quercus sp., 10 May 2009, GK3799 (ACAM 2010-128), coll. Ch. Chrysopoulos and K. Giatra. Trikala Prefecture: Koziakas Mt., in soil under mixed forest of Quercus sp. and P. nigra, 2 April 2013, GK6538 (ACAM 2013-073), coll. K. Papadimitriou (GenBank: MK113978); Koziakas Mt., in soil under mixed forest of Quercus sp. and P. nigra, 2 April 2013, GK6537 (ACAM 2013-074), coll. K. Papadimitriou. Ioannina Prefecture: Metsovo, in soil under pure stand of Q. coccifera, 18 April 2016, GK9408 (ACAM 2016001), coll. A. Bideris (GenBank: MK113977); Metsovo, in soil under pure stand of $Q$. coccifera, 19 April 2016, GK9409 (ACAM 2016-002), coll. A. Bideris; Metsovo, in soil under pure stand of Q. coccifera, 19 April 2016, GK9410 (ACAM 2016-003), coll. A. Bideris; Demati, in soil under pure stand of Q. coccifera, 22 March 2017, GK10231 (ACAM 2017-033), coll. A. Bideris. Attica Prefecture: Katsimidi, in soil under mixed forest of Q. ilex and P. halepensis, 22 March 2016, VK4482 (ACAM 2016-004), coll. V. Kaounas (GenBank: MK113980); Katsimidi, in soil under mixed forest of $Q$. ilex and P. halepensis, 12 April 2016, VK4506 (ACAM 2016-005), coll. V. Kaounas (GenBank: MK113980). Ioannina Prefecture: Neochoropoulo, in soil under a mixed stand of $Q$. coccifera and Q. ilex, 27 April 2016, LT1183 (ACAM 2016-006), coll. V. Nakkas (GenBank: MK113976). BULGARIA. Varna, Dolishte village, in soil under pure stand of Carpinus sp., 07 June 2017, MSL 1945 F8517 (SOMF 29978; ACAM 2017-034), coll. R. Radev (GenBank: MK113981). Sliven, in soil under a mixed stand of Quercus robur, 


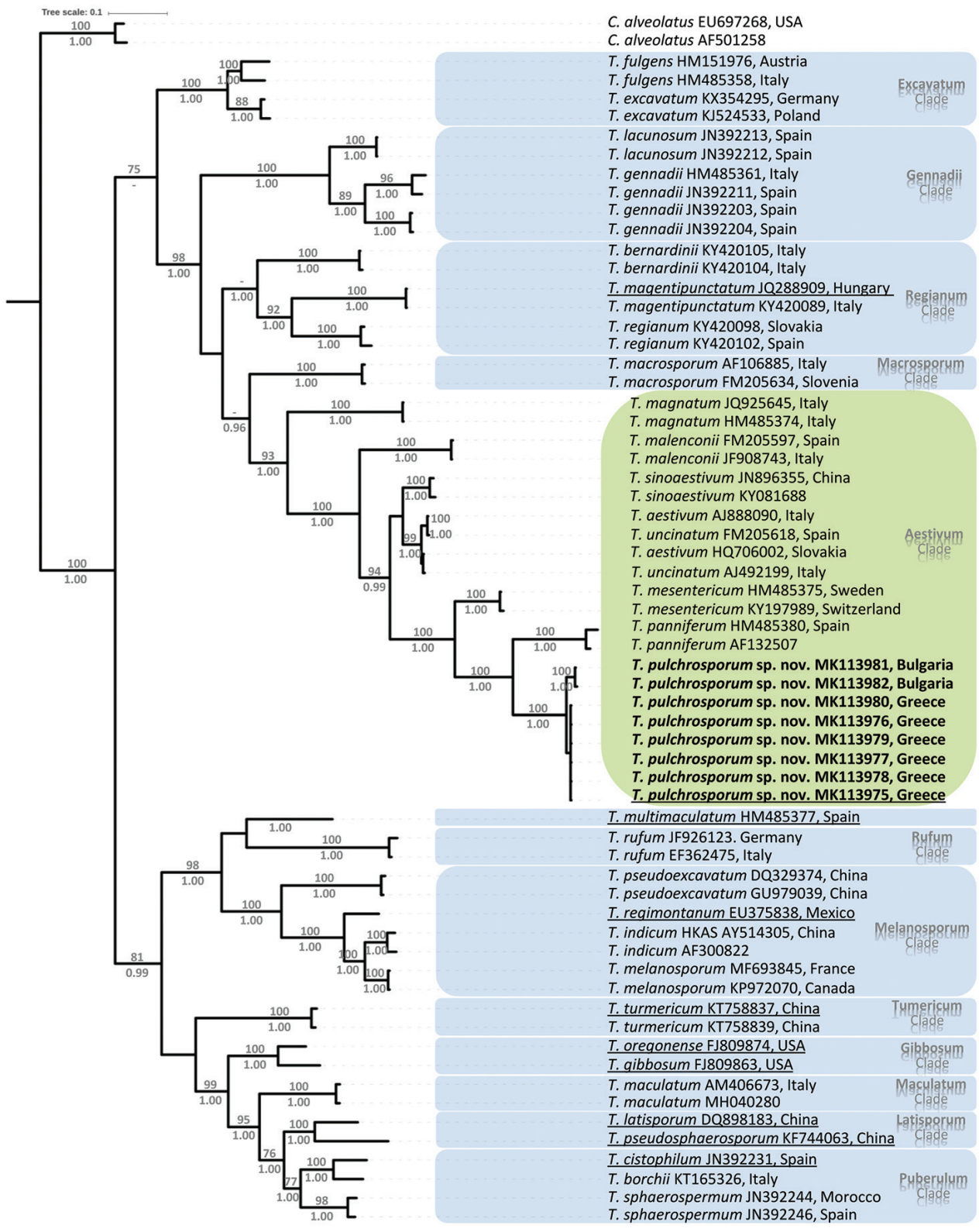

Figure 5. Phylogenetic tree inferred from Bayesian analysis including 62 ITS sequences assigned to 31 Tuber taxa, including members of major clades of the genus. Sequences are labelled with Latin binomials, GenBank accession numbers and geographic origin. T. pulchrosporum sp. nov. is indicated in boldface. Reference sequences deriving from type material are underlined. Choiromyces alveolatus (Tuberaceae) was used as the outgroup. Bootstrap (BS) values from Maximum Likelihood (ML) analysis ( $\geq 70 \%)$ and Posterior Probabilities (PPs) from Bayesian Inference $(\geq 0.95)$ are shown at the nodes of branches. 
Corylus sp., Carpinus sp. and Acersp., 09 August 2017, MSL 1961 F0388 (SOMF 29979; ACAM 2017-035), coll. K. Pilasheva \& P. Neikov (GenBank: MK113982).

Phylogenetic aspects. The resultant ITS sequence data comprises of 64 sequences which were aligned at 780 sites, 738 of which represent the ITS1-5.8S-ITS2 region, i.e. between the end of the SSU motif (CATTA) and the beginning of LSU motif (TAGGG) (Bonito et al. 2010a). ML and BI analyses yielded similar tree topologies and only the tree inferred from the Bayesian analysis is presented (Fig. 5). The morphologically variable genus Tuber is monophyletic (BS: 100\%, PP: 1.00) and several lineages are revealed; for the purposes of this study, the following highly supported clades were included: Aestivum, Excavatum, Gennadii, Gibbosum, Latisporum, Maculatum, Macrosporum, Melanosporum, Puberulum, Regianum, Rufum, Tumericum (=Japonicum).

According to the phylogenetic analysis performed, T. pulchrosporum belongs to the Aestivum clade. All eight sequences of this new taxon form a distinct highly supported subclade (BS: 100\%, PP: 1.00). Greek specimens possessed almost identical ITS sequences $(99.8-100 \%)$ and so did Bulgarian samples, whereas the comparison between collections from the two countries resulted in sequence identity values of $98.13 \pm$ $0.08 \%$. In total, intraspecific sequence identity values for $T$. pulchrosporum exceeded 98\% (i.e. $98.05-100 \%$ ). The new species is sister to T. panniferum (BS: 100\%, PP: $1.00)$; the respective sequences demonstrated low sequence identity $(73.21-75.08 \%)$ further evidencing their distinct taxonomic status.

\section{Discussion}

The molecular analysis evidenced that the eight sequences representing T.pulchrosporum are grouped within the Aestivum clade by forming a distinct terminal group supported with high BS and PP values. The closest phylogenetic relative of T. pulchrosporum is T. panniferum Tul. \& C. Tul., i.e. a Mediterranean species with analogous ecological preferences (Jeandroz et al. 2008). T. panniferum also exhibits a rather similar macromorphology characterised by a brownish pubescent peridium, absence of pyramidal warts and ascomata often bearing a cavity, although the tomentum is much more prominent, exhibiting thus a felted appearance. However, the microscopic features of the two species are clearly different. In T. panniferum, the ornamentation consists of isolated spines never exceeding $3 \mu \mathrm{m}$ in height, while the peridial surface is covered by rusty brown hyphae which form a dense cottony mass (Montecchi and Sarasini 2000; Riousset et al. 2001; Moreno-Arroyo et al. 2005).

By morphology alone, T. pulchrosporum is easily distinguishable within the Aestivum clade since no other species produces ascospores bearing such a uniquely crested ornamentation. The more distant T. aestivum (Wulfen) Spreng. (including T. uncinatum Chatin) and T. sinoaestivum J.P. Zhang \& P.G. Liu could be distinguished macroscopically thanks to their blackish peridial surface with prominent pyramidal warts and ascospores bearing a complete reticulum. Ascospores of T. mesentericum Vittad. show some affinity in their outline to those of $T$. pulchrosporum but they clearly possess a much more reticulate network; moreover, the peridial surface is black with pyramidal warts as in T. aestivum. 
Although phylogenetically more distant, some other species with asci containing 1-8 ascospores may superficially resemble T. pulchrosporum. Hence, T. regianum Montecchi \& Lazzari, the recently described T. magentipunctatum Z. Merényi, I. Nagy, Stielow \& Bratek and T. bernardinii Gori, all belonging to the Regianum clade (Zambonelli et al. 2016; Crous et al. 2017), possess a reddish-brown to brown peridial surface with dense and rather flat warts as in the case of T. pulchrosporum. However, they all produce ascospores with pointed spines which are connected to form a complete reticulum. Ascomata of T. malenconii Donadini, Riousset, G. Riousset \& G. Chev and T. pseudoexcavatum Y. Wang, G. Moreno, Riousset, Manjón \& G. Riousset also show a macroscopic resemblance to T. pulchrosporum, with their rough indistinctly warty peridial surface (black for the former and brown for the latter), often with a similar basal cavity as well. However, ascospores of both T. malenconii and T. pseudoexcavatum have short spines, basally/broadly connected, exhibiting a more or less regular reticulum (Donadini et al. 1979; Manjón et al. 2009). Therefore, the unique type of ornamentation of $T$. pulchrosporum ascospores clearly distinguishes it from all species with similar macroscopic appearance.

\section{Acknowledgements}

This work was partly financed by the IBER-BAS project "Taxonomy, conservation and sustainable use of fungi" and by research funding provided to GZ. The following individuals are acknowledged for kindly providing truffle specimens for examination: A. Aggelopoulou, A. Bideris, Ch. Chrysopoulos, K. Giatra, P. Neikov, P. Panagiotidis, K. Papadimitiriou, K. Pilasheva and R. Radev.

\section{References}

Agnello C, Kaounas V (2011) Tuber asa and T. gennadii. A close morphological study of two species often confused in the past with a brief historical bibliographic summary. Ascomecete.org 3: 65-74.

Alvarado P, Moreno G, Manjón J (2012a) Comparison between Tuber gennadii and T. oligospermum lineages reveals the existence of the new species T. cistophilum (Tuberaceae, Pezizales). Mycologia 104: 894-910. https://doi.org/10.3852/11-254

Alvarado P, Moreno G, Manjón J (2012b) A new Tuber without spore ornamentation, Tuber melosporum comb. nov. Boletín de la Sociedad Micológica de Madrid 36: 194.

Assyov B, Slavova M (2018) New records of Bulgarian hypogeous fungi. $11^{\text {th }}$ Seminar of Ecology, 26-27 April 2018, Sofia, Bulgaria, Book of Abstracts, IBER-BAS, Sofia, 28.

Belfiori B, Riccioni C, Paolocci F, Rubini A (2016) Characterization of the reproductive mode and life cycle of the whitish truffle T. borchii. Mycorrhiza 26: 515-527. https://doi. org/10.1007/s00572-016-0689-0

Berch SM, Bonito G (2016) Truffle diversity (Tuber, Tuberaceae) in British Columbia. Mycorrhiza 26: 587-594. https://doi.org/10.1007/s00572-016-0695-2 
Bonito G, Gryganskyi AP, Trappe JM, Vilgalys R (2010a) A global meta-analysis of Tuber ITS rDNA sequences: species diversity, host associations and long-distance dispersal. Molecular Ecology 19: 4994-5008. https://doi.org/10.1111/j.1365-294X.2010.04855.x

Bonito G, Smith ME, Nowak M et al. (2013) Historical biogeography and diversification of truffles in the Tuberaceae and their newly identified Southern Hemisphere sister lineage. PLoS ONE 8(1): e52765. https://doi.org/10.1371/journal.pone.0052765

Bonito G, Trappe JM, Rawlinson P, Vilgalys R (2010b) Improved resolution of major clades within Tuber and taxonomy of species within the Tuber gibbosum complex. Mycologia 102: 1042-1057. https://doi.org/10.3852/09-213

Ceruti A, Fontana A, Nosenzo C (2003) Le specie europee del genere Tuber. Una revisione storica. Museo Resionale di Scienze Naturali, Torino.

Chen J, Guo SX, Liu PG (2011) Species recognition and cryptic species in the Tuber indicum complex. PLoS ONE 6(1): e14625. https://doi.org/10.1371/journal.pone.0014625

Chen J, Liu PG (2007) Tuber latisporum sp. nov. and related taxa, based on morphology and DNA sequence data. Mycologia 99: 475-481. https://doi.org/10.1080/15572536.2007.1 1832572

Crous PW, Wingfield MJ, Burgess TI et al. (2017) Tuber magentipunctatum Z. Merényi, I. Nagy, Stielow \& Bratek, sp. nov. Fungal Planet description sheets: 558-624, Persoonia 38: 240-384. https://doi.org/10.3767/003158517X698941

Diamandis S, Perlerou C (2008) Recent records of hypogeous fungi in Greece. Acta Mycologica 43: 139-142. https://doi.org/10.5586/am.2008.017

Dimitrova E, Gyosheva M (2008) Hypogeous ascomycetes in Bulgaria. Phytologia Balcanica 14: 309-314.

Donadini JC, Riousset L, Riousset G, Chevalier G (1979) Tuber malençoni nov. sp. Bulletin de la Société mycologique de France 94: 351-358.

El Karkouri K, Murat C, Zampieri E, Bonfante P (2007) Identification of internal transcribed spacer sequence motifs in truffles: a first step toward their DNA bar coding. Applied and Environmental Microbiology 73: 5320-5330. https://doi.org/10.1128/AEM.00530-07

Fan L, Liu X, Cao J (2015) Tuber turmericum sp. nov., a Chinese truffle species based on morphological and molecular data. Mycological Progress 14: 111. https://doi.org/10.1007/ s11557-015-1134-z

Fan L, Yue SF (2013) Phylogenetic divergence of three morphologically similar truffles, Tuber sphaerosporum, T. sinosphaerosporum, and T. pseudosphaerosporum sp. nov. Mycotaxon 125: 283-288. https://doi.org/10.5248/125.283

Ferdman Y, Aviram S, Roth-Bejerano N, Trappe JM, Kagan-Zur V (2005) Phylogenetic studies of Terfezia pfeilii and Choiromyces echinulatus (Pezizales) support new genera for southern African truffles: Kalaharituber and Eremiomyces. Mycological Research 109: 237-245. https://doi.org/10.1017/S0953756204001789

Gryndler M, Hršelová H, Soukupová L, Streiblová E, Valda S, Borovička J, Gryndlerová H, Gazo J, Miko (2011) Detection of summer truffle (Tuber aestivum Vittad.) in ectomycorrhizae and in soil using specific primers. FEMS Microbiology Letters 318: 84-91. https:// doi.org/10.1111/j.1574-6968.2011.02243.x

Guevara G, Bonito G, Cazares E, Rodriguez JA, Trappe J, Vilgalys R (2008) Tuber regimontanum, new species of truffle from Mexico. Revista Mexicana de Micología 26: 17-20. 
Guevara-Guerrero G, Bonito G, Smith ME, Healy R, Grupe AC II, Cázares E, Castellano MA, Trappe JM (2018) Tuber aztecorum sp. nov., a truffle species from Mexico belonging to the Maculatum clade (Tuberaceae, Pezizales). MycoKeys 28: 61-72. https://doi.org/10.3897/ mycokeys.30.22887

Gyosheva M, Assyov B, Konstantinidis G, Stoykov D (2012) Collections of Tuber macrosporum from the Balkan Peninsula (Bulgaria and Greece). Ascomycete.org 4: 75-78.

Hanson AM, Hodge K, Porter LM (2003) Mycophagy among primates. Mycologist 17: 6-10. https://doi.org/10.1017/S0269915X0300106X

Iotti M, Amicucci A, Bonito G, Bonuso E, Stocchi V, Zambonelli A (2007) Selection of a set of specific primers for the identification of Tuber rufum: a truffle species with high genetic variability. FEMS Microbiology Letters 277 (2): 223-231. https://doi.org/10.1111/j.15746968.2007.00963.x

Jeandroz S, Murat C, Wang Y, Bonfante P, Tacon FL (2008) Molecular phylogeny and historical biogeography of the genus Tuber, the 'true truffles'. Journal of Biogeography 35(5): 815-829. https://doi.org/10.1111/j.1365-2699.2007.01851.x

Katoh K, Standley DM (2013) MAFFT multiple sequence alignment software version 7: improvements in performance and usability. Molecular Biology and Evolution 30: 772-780. https://doi.org/10.1093/molbev/mst010

Konstantinidis G (2009) Mushrooms, a photographic collector's guide. Published by the author, Athens, Greece. [in Greek]

Kumar S, Stecher G, Li M, Knyaz C, Tamura K (2018) MEGA X: Molecular Evolutionary Genetics Analysis across Computing Platforms. Molecular Biology and Evolution 35: 1547-1549. https://doi.org/10.1093/molbev/msy096

Lacheva M (2012) New records for hypogeous ascomycetes in Bulgaria. Science and Technologies 2: 30-34.

Letunic I, Bork P (2016) Interactive tree of life (iTOL) v3: an online tool for the display and annotation of phylogenetic and other trees. Nucleic Acids Research 44(W1): W242-5. https://doi.org/10.1093/nar/gkw290

Mabru D, Dupré C, Douet JP, Leroy P, Ravel C, Ricard JM, Médina B, Castroviejo M, Chevalier G (2001) Rapid molecular typing method for the reliable detection of Asiatic black truffle (Tuber indicum) in commercialized products: fruiting bodies and mycorrhizal seedlings. Mycorrhiza 11: 89-94. https://doi.org/10.1007/s005720100103

Manjón JL, García-Montero LG, Alvarado P, Moreno G, Di Massimo G (2009) Tuber pseudoexcavatum versus $T$. pseudohimalayense - new data on the molecular taxonomy and mycorrhizae of Chinese truffles. Mycotaxon 110: 399-412. https://doi.org/10.5248/110.399

Marjanović Ž, Grebenc T, Marković M, Glišić A, Milenković M (2010) Molecular diversity and ecological specificity of truffles (genus Tuber) originating from mid-west of the Balkan Peninsula. Sydowia 62: 67-87.

Mello A, Cantisani A, Vizzini A, Bonfante P (2002) Genetic variability of Tuber uncinatum and its relatedness to other black truffles. FEMS Microbiology Letters 4: 584-594.

Mello A, Murat C, Bonfante P (2006) Truffles: Much more than a prized and local fungal delicacy. FEMS Microbiology Letters 260: 1-8. https://doi.org/10.1111/j.1574-6968.2006.00252.x

Milenković M, Grebenc T, Marković M, Ivančević B (2015) Tuber petrophilum, a new species from Serbia. Mycotaxon 130: 1141-1152. https://doi.org/10.5248/130.1141 
Miller MA (2010) CIPRES Science Gateway survey results. http://www.phylo.org/tools/ survey2.html

Montecchi A, Sarasini M (2000) Fungi ipogei d'Europa. Centro studi Micologici. Associazione Micologica Bresadola, Trento.

Moreno-Arroyo B, Gómez-Fernández J, Pulido-Calmaestra E (2005) Tesoros de Nuestros Montes. Trufas de Andalucía. Consejería de Medio Ambiente, Junta de Andalucía, Córdoba.

Nedelin T, Gyosheva M, Kostov K, Savev K (2016) New records and data on hypogeous ectomycorrhizal fungi in Bulgaria. Forestry Ideas 22: 113-126.

Nylander JAA (2004) MrModeltest v2. Program distributed by the author. Evolutionary Biology Centre, Uppsala University.

Okonechnikov K, Golosova O, Fursov M (2012) Unipro UGENE: a unified bioinformatics toolkit. Bioinformatics 28: 1166-1167. https://doi.org/10.1093/bioinformatics/bts091

Osmundson TW, Robert VA, Schoch CL, Baker LJ, Smith A, Robich G, Mizzan L, Garbelotto MM (2013) Filling gaps in biodiversity knowledge for macrofungi: contributions and assessment of an herbarium collection DNA barcode sequencing project. PLoS ONE 8: e62419. https://doi.org/10.1371/journal.pone.0062419

Pacioni G (1984) Hysterangium epiroticum nov. sp. and other hypogeous macromycetes from S.S.R. (Albania). Nova Hedwigia 40: 79-84.

Payen T, Murat C, Bonito G (2014) Truffle phylogenomics: new insights into truffle evolution and truffle life cycle. In Fungi, vol. 70, Advances in Botanical Research. Academic Press Elsevier, 488 p.

Piña Páez C, Bonito GM, Guevara-Guerrero G, Castellano MA, Garibay-Orijel R, Trappe JM, Peña Rámirez R (2018) Description and distribution of Tuber incognitum sp. nov. and Tuber anniae in the Transmexican Volcanic Belt. MycoKeys 41: 17-27. https://doi. org/10.3897/mycokeys.41.28130

Riousset LG, Chevalier G, Bardet MC (2001) Truffles d'Europe et de Chine. INRA, Paris.

Ronquist F, Teslenko M, van der Mark P, Ayres DL, Darling A, Höhna S, Larget B, Liu L, Suchard MA, Huelsenbeck JP (2012) MrBayes 3.2: Efficient Bayesian phylogenetic inference and model choice across a large model space. Systematic Biology 61: 539-542. https://doi.org/10.1093/sysbio/sys029

Roux C, Sejalon-Delmas N, Martins M, Parguey-Leduc A, Dargent R, Becard G (1999) Phylogenetic relationships between European and Chinese truffles based on parsimony and distance analysis of ITS sequences. FEMS Microbiology Letters 180: 147-155. https://doi. org/10.1111/j.1574-6968.1999.tb08789.x

Royal Botanic Garden Edinburgh (1969) Flora of British fungi: colour identification chart. H.M. Stationery Office, Edinburgh.

Schickmann S, Urban A, Kräutler K, Nopp-Mayr U, Hackländer K (2012) The interrelationship of mycophagous small mammals and ectomycorrhizal fungi in primeval, disturbed and managed Central European mountainous forests. Oecologia 170: 395-409. https:// doi.org/10.1007/s00442-012-2303-2

Schiebold JMI, Bidartondo MI, Karasch P, Gravendeel B, Gebauer G (2017) You are what you get from your fungi: nitrogen stable isotope patterns in Epipactis species. Annals of Botany 119: 1085-1095. https://doi.org/10.1093/aob/mcw265 
Schneider-Maunoury L, Leclercq S, Clement C, Coves H, Lambourdiere J, Sauve M, Richard F, Selosse M-A, Taschen E (2018) Is Tuber melanosporum colonizing the roots of herbaceous, non-ectomycorrhizal plants? Fungal Ecology 31: 59-68. https://doi.org/10.1016/j. funeco.2017.10.004

Selosse MA, Faccio A, Scappaticci G, Bonfante P (2004) Chlorophyllous and achlorophyllous specimens of Epipactis microphylla (Neottieae, Orchidaceae) are associated with ectomycorrhizal septomycetes, including truffles. Microbial Ecology 47: 416-426. https://doi. org/10.1007/s00248-003-2034-3

Sievers F, Higgins DG (2018) Clustal Omega for making accurate alignments of many protein sequences. Protein Science 27: 135-145. https://doi.org/10.1002/pro.3290

Stamatakis A (2014) RAxML version 8: a tool for phylogenetic analysis and post-analysis of large phylogenies. Bioinformatics 30: 1312-1313. https://doi.org/10.1093/bioinformatics/btu033

Stobbe U, Buntgen U, Sproll L, Tegel W, Egli S, Fink S (2012) Spatial distribution and ecological variation of re-discovered German truffle habitats. Fungal Ecology 5: 591-599. https:// doi.org/10.1016/j.funeco.2012.02.001

Trappe JM, Claridge A (2010) The hidden life of truffles: not just for gourmands, truffles play essential roles in the health of ecosystems. Scientific American 302: 78-84. https://doi. org/10.1038/scientificamerican0410-78

Trappe JM, Molina R, Luoma DL, Cázares E, Pilz D, Smith JE, Castellano MA, Miller L, Trappe MJ (2009) Diversity, ecology and conservation of the truffle fungi in forests of the Pacific northwest. US Dept. of Agriculture, Forest Service General Technical Report PNW-GTR-772. https://doi.org/10.2737/PNW-GTR-772

Wang Y, Tan ZM, Zhang DC, Murat C, Jeandroz S, Le Tacon F (2006) Phylogenetic relationships between Tuber pseudoexcavatum, a Chinese truffle, and other Tuber species based on parsimony and distance analysis of four different gene sequences. FEMS Microbiology Letters: 259: 269-281. https://doi.org/10.1111/j.1574-6968.2006.00283.x

Weden C (2005) Black truffles of Sweden. Systematics, population studies, ecology and cultivation of Tuber aestivum syn. T. uncinatum. Department of Systematic Botany, Uppsala University, Uppsala.

White TJ, Bruns T, Lee S, Taylor JW (1990) Amplification and direct sequencing of fungal ribosomal RNA genes for phylogenetics. In: Innis MA, Gelfand DH, Sninsky JJ, White TJ (Eds) PCR Protocols: a guide to methods and applications. Academic Press Inc., New York, 315-322. https://doi.org/10.1016/B978-0-12-372180-8.50042-1

Zambonelli A, Iotti M, Murat C, (2016) True Truffle (Tuber spp.) in the World. Soil Biology, vol 47. Springer, Switzerland. https://doi.org/10.1007/978-3-319-31436-5

Zervakis G, Lizoň P, Dimou D, Polemis E (1999) Annotated check-list of the Greek macrofungi. II. Ascomycotina. Mycotaxon 72: 487-506.

Zhang JP, Liu PG, Chen J (2012) Tuber sinoaestivum sp. nov., an edible truffle from southwestern China. Mycotaxon 122: 73-82. https://doi.org/10.5248/122.73

Zhang LF, Yang Z, Song DS (2005) A phylogenetic study of commercial Chinese truffles and their allies: taxonomic implications. FEMS Microbiology Letters 245: 85-92. https://doi. org/10.1016/j.femsle.2005.02.028 